\title{
Modeling micelle formation and interfacial properties with iSAFT classical density functional theory
}

\author{
Le Wang, ${ }^{\text {a) }}$ Amin Haghmoradi, Jinlu Liu, Shun Xi, George J. Hirasaki, Clarence A. Miller, \\ and Walter G. Chapman ${ }^{\text {b) }}$ \\ Department of Chemical and Biomolecular Engineering, Rice University, 6100 Main St., \\ Houston, Texas 77005, USA
}

(Received 3 October 2016; accepted 1 March 2017; published online 29 March 2017)

\begin{abstract}
Surfactants reduce the interfacial tension between phases, making them an important additive in a number of industrial and commercial applications from enhanced oil recovery to personal care products (e.g., shampoo and detergents). To help obtain a better understanding of the dependence of surfactant properties on molecular structure, a classical density functional theory, also known as interfacial statistical associating fluid theory, has been applied to study the effects of surfactant architecture on micelle formation and interfacial properties for model nonionic surfactant/water/oil systems. In this approach, hydrogen bonding is explicitly included. To minimize the free energy, the system minimizes interactions between hydrophobic components and hydrophilic components with water molecules hydrating the surfactant head group. The theory predicts micellar structure, effects of surfactant architecture on critical micelle concentration, aggregation number, and interfacial tension isotherm of surfactant/water systems in qualitative agreement with experimental data. Furthermore, this model is applied to study swollen micelles and reverse swollen micelles that are necessary to understand the formation of a middle-phase microemulsion. Published by AIP Publishing. [http://dx.doi.org/10.1063/1.4978503]
\end{abstract}

\section{INTRODUCTION}

Surfactants play important roles in commercial and industrial applications, such as personal care products and enhanced oil recovery (EOR). ${ }^{1,2}$ EOR has emerged during past decades as a method to recover oil trapped in the reservoir rock after water flooding. As an EOR technique, surfactant flooding involves injection of surfactants into a reservoir to reduce the interfacial tension between the oil and water phases. At lower interfacial tension, crude oil trapped by capillary effects becomes mobile and can more easily be displaced by injected fluids, which results in higher production. Researchers have found that ultralow interfacial tension (below $10^{-3} \mathrm{mN} / \mathrm{m}$ ) usually appears with a thermodynamically stable middle-phase microemulsion. By optimizing surfactant architecture according to reservoir composition and conditions (temperature, pressure, salinity, etc.), the middle-phase microemulsion can be obtained, reaching ultralow interfacial tension and increasing oil recovery efficiency.

Winsor ${ }^{3}$ proposed the R-ratio theory to describe the phase behavior of oil/water/surfactant systems. The R-ratio is defined as the ratio of energies of interaction between surfactant layer and oil-rich phase to that between surfactant layer and waterrich phase. For a planar surfactant layer, if the interaction between surfactants and oil phase is more favorable, corresponding to $R>1$, the surfactant layer would tend to increase the area of contact with the oil phase and bend toward the

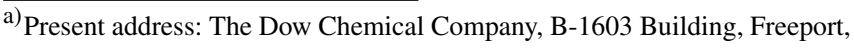
Texas 77541, USA

b) wgchap@ rice.edu
}

water phase. Hence, oil would tend to be the continuous phase and a water-in-oil microemulsion is favored. If the interaction between surfactant layer and water phase is greater, which corresponds to $R<1$, water tends to become the continuous phase and an oil-in-water microemulsion is favored. Otherwise, if the interaction energies are the same, which corresponds to $R=1$, a middle-phase microemulsion is favorable. The R-ratio can also be viewed as the tendency of surfactant layer to become convex toward the oil phase over the tendency of surfactant layer to become convex toward the water phase. Following Winsor's pioneering work, Fraaije $e t$ al ${ }^{4}$ recently proposed an analogous but more quantified method to predict the formation of a microemulsion, referred to as method of moments. In this model, the stress profile across a planar interface is calculated, based on which the surface torque density is examined. The surface torque density can be interpreted as the tendency of surfactant layer to become convex toward oil minus the tendency of surfactant layer to become convex toward water, similar to the Winsor R-ratio. Thus, it was shown that an Rratio equal to 1 is equivalent to surface torque density equal to 0 , at which condition a middle-phase microemulsion is favorable. In the work of Fraaije et al., the stress profile was calculated from Dissipative Particle Dynamics (DPD). ${ }^{5}$ The stress profile can also be calculated from Molecular Dynamics (MD) simulation ${ }^{6}$ or classical Density Functional Theory (DFT). ${ }^{7}$ The method of moments quantitatively bridges the gap between surfactant architecture and their bulk phase behavior, but it requires that the interfacial tension is nearly zero. The ability of surfactant to acquire ultralow interfacial tension is determined by its efficiency at the oil-water interface, which is influenced by the equilibrium among micelles, 
surfactant monomers in solution, and surfactant at the interface. To correctly calculate interfacial tension and apply the method of moments, a model must capture this equilibrium.

Two approaches are most commonly applied to predict the critical micelle concentration (CMC). ${ }^{8}$ One is the pseudophase approach, in which the micelles are assumed to be a surfactant rich phase that is in equilibrium with surfactant monomers. By assuming a homogeneous distribution of surfactant molecules or surfactant tails in the surfactant rich phase, the CMC can be calculated from bulk theories such as Statistical Associating Fluid Theory (SAFT) equation of state. ${ }^{9}$ However, since the distribution of surfactant tails and other components inside a micelle is not uniform, this approach may prove inaccurate. The other approach is the massaction approach, ${ }^{10}$ which considers the equilibrium between micelles with different sizes and shapes (instead of a homogeneous phase) and surfactant monomers. Inside this framework, a molecular-thermodynamic approach introduced by Nagrajan and Ruckenstein, ${ }^{11}$ Puvvada and Blankschtein, ${ }^{12-14}$ and Lukanov and Firoozabadi ${ }^{15}$ has been shown to successfully predict thermodynamic properties such as CMC and micelle size distribution. Despite great success in predicting $\mathrm{CMC}$, theories able to predict the micelle formation, the distribution of molecules inside a micelle, and interfacial properties such as interfacial tension in a unified fashion are rare to find, which contributes to part of our motivation.

Recently, models based on statistical mechanics such as classical density functional theory (DFT) have been developed to model the meso-scale structure of complex fluids. DFT has shown its strength in modeling inhomogeneous and complex fluids and excellent agreement with molecular simulations and experiments for a variety of systems, including the phase behavior of associating fluids under confinement, ${ }^{65,66}$ the behavior of polymer brushes, ${ }^{17-21}$ the phase behavior and structure of block copolymers, ${ }^{22-24}$ the interfacial properties of oil/water systems, ${ }^{25,26}$ and the impact of surfactant architecture on interfacial properties. ${ }^{27}$ The theory can be computationally more efficient than molecular simulations since density fields rather than trajectories of individual molecules are calculated, and the method takes advantage of system symmetry. More importantly, due to the limitation of computing resources, molecular simulation cannot model true thermodynamic equilibrium between surfactant monomers and aggregates. Because low surfactant monomer concentration in the bulk would result in large numbers of solvent molecules in the simulation box, simulations cannot be efficiently conducted within reasonable period of time, while DFT does not have this issue. These advantages make DFT a more suitable approach in modeling micelle formation and surfactant phase behavior compared with simulation techniques.

The goal of this work is to demonstrate the feasibility of applying interfacial Statistical Associating Fluid Theory (iSAFT) $\mathrm{DFT}^{28,29}$ to predict the CMC and describe micelle/reverse micelle structure for model nonionic surfactants, and to establish the theoretical basis for molecular understanding of the impact of surfactant architecture on surfactant phase behavior. In this work, we apply iSAFT to investigate the effect of surfactant architecture on the CMC, the micellar structure, aggregation number, and interfacial tension. Furthermore, the formation of swollen micelle and reverse swollen micelle are also studied. To our knowledge, this is the first time DFT is applied to model such systems.

\section{THEORETICAL BACKGROUND}

In Secs. II A and II B, details of the potential models and iSAFT DFT are described.

\section{A. Potential models}

The Statistical Associating Fluid Theory (SAFT) ${ }^{30}$ has shown great success in modeling the phase behavior of polyatomic associating fluids. ${ }^{67}$ In this approach, molecules are modeled as chains of segments. All the segments are spherical with hard sphere diameter $\sigma$ that also interact through a Lennard-Jones potential and directional hydrogen bonding sites. As the generalization of the SAFT, interfacial Statistical Associating Fluid Theory (iSAFT) allows the prediction of interfacial properties ${ }^{31,32}$ and meso-scale structure of complex fluids. In the bulk, iSAFT reduces to the SAFT form.

The segment-segment hard sphere repulsion and longrange attraction are modeled with the pair potential,

$$
u_{\alpha \beta}(r)= \begin{cases}\infty & r<\sigma_{\alpha \beta}, \\ u_{\alpha \beta}^{L J}\left(r_{\min }\right)-u_{\alpha \beta}^{L J}\left(r_{c}\right) & \sigma_{\alpha \beta} \leq r<r_{\min }, \\ u_{\alpha \beta}^{L J}(r)-u_{\alpha \beta}^{L J}\left(r_{c}\right) & r_{\min } \leq r<r_{c}, \\ 0 & r \geq r_{c},\end{cases}
$$

where $u_{\alpha \beta}^{L J}$ represents the Lennard-Jones potential, $r$ is the distance between segment $\alpha$ and $\beta, \sigma_{\alpha \beta}$ is the cross species hard sphere diameter determined by specific mixing rules, $r_{\text {min }}$ represents the Lennard-Jones potential minimum, and $r_{c}$ denotes the cutoff radius which is set to $4 \sigma_{\alpha \beta}$.

The Lennard-Jones potential $u_{\alpha \beta}^{L J}$ is defined as

$$
u_{\alpha \beta}^{L J}(r)=4 \varepsilon_{\alpha \beta}\left[\left(\frac{\sigma_{\alpha \beta}}{r}\right)^{12}-\left(\frac{\sigma_{\alpha \beta}}{r}\right)^{6}\right]
$$

where $\varepsilon_{\alpha \beta}$ is the depth of attraction well between segment $\alpha$ and $\beta$, which is determined by specific mixing rules. In this work, cross interaction parameters for Lennard-Jones interactions of two unlike segments $\alpha$ and $\beta$ are given by

$$
\varepsilon_{\alpha \beta}=\sqrt{\varepsilon_{\alpha} \varepsilon_{\beta}}\left(1-k_{\alpha \beta}\right), \quad \sigma_{\alpha \beta}=\frac{\sigma_{\alpha}+\sigma_{\beta}}{2},
$$

where $\varepsilon_{\alpha}$ and $\varepsilon_{\beta}$ are Lennard-Jones interaction energies of segments $\alpha$ and $\beta$, respectively; while $\sigma_{\alpha}$ and $\sigma_{\beta}$ are diameters of segments $\alpha$ and $\beta$, respectively. $k_{\alpha \beta}$ is the binary interaction parameter for the two segments.

Molecules such as water and surfactants may form hydrogen bonds. The association between molecules is described by a directional attractive potential between association sites as shown in Figure 1. An orientationally dependent square well potential is used to model the hydrogen bonding interaction energy given by

$$
u_{A B}^{a s s o c}\left(r, \Omega_{\alpha}, \Omega_{\beta}\right)=\left\{\begin{array}{ll}
-\varepsilon_{\text {assoc }} & r<r_{c, \text { assoc }} ; \theta_{A}<\theta_{c} ; \theta_{B}<\theta_{c}, \\
0 & \text { otherwise }
\end{array},\right.
$$




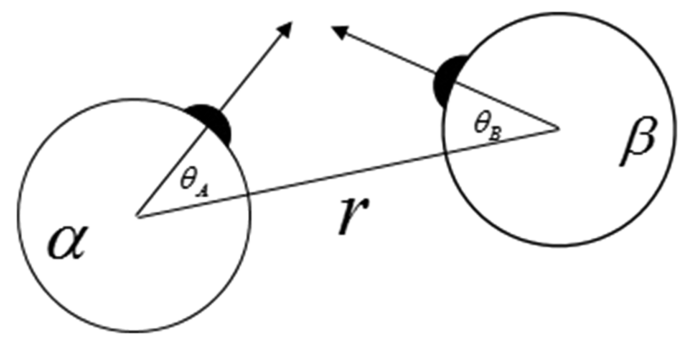

FIG. 1. Potential model for association.

where $\varepsilon_{a s s o c}$ denotes the association energy between association site $A$ on segment $\alpha$ and association site $B$ on segment $\beta . r_{c, a s s o c}$ is the cutoff distance within which association can occur. $\Omega_{\alpha}$ and $\Omega_{\beta}$ are the orientations of segments $\alpha$ and $\beta$, respectively. $\theta$ is the angle between the vector pointing from the center of a segment to the center of the association site on that segment and the vector pointing from the center of one segment ( $\alpha$ or $\beta$ ) to the center of the other segment ( $\beta$ or $\alpha$ ). Association can only occur when these two angles are smaller than the cutoff value $\theta_{c}$ (Figure 1). The cutoff distance and the angular cutoffs are chosen to allow only one bond per association site.

In this work, potential parameters for water and oil are fit to saturated liquid densities and vapor pressures. The surfactant is constructed in the same spirit as the Telo de Gama and Gubbins model ${ }^{33}$ where the surfactants include segments with water-like parameters and segments with alkane-like parameters. Here, we explicitly include hydrogen bonding which was not present in Telo de Gama and Gubbins model. The schematics of model water, octane, and surfactant molecules are shown in Figure 2. For example, water is modeled as a sphere with four association sites: two electron donor and two electron acceptor sites. Fitting of the model parameters is described in Sec. III.

\section{B. Interfacial statistical associating fluid theory}

Based on the potential models, the iSAFT free energy functional of the system $A$ can be constructed. In the framework of thermodynamic perturbation theory, $A$ is given by the summation of various contributions, i.e., ideal gas contribution $A^{i d}$, hard sphere contribution $A^{\text {hs }}$, long range attraction contribution $A^{\text {att }}$, association contribution $A^{\text {assoc }}$, and contribution due to the covalent bond between segments to form

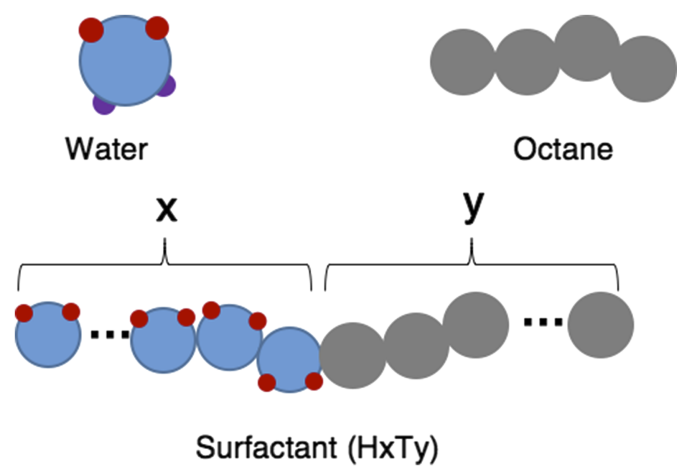

FIG. 2. Schematics of water, octane, and amphiphilic chain constructed within the Telo da Gama and Gubbins model. chain-like molecules $A^{\text {chain }}$. In the following, we review expressions of Helmholtz free energy due to these different contributions. More details can be found in the original publications by Jain et al. ${ }^{29}$ and Bymaster and Chapman. ${ }^{34}$

The ideal gas contribution for a mixture of spheres is exactly known from statistical mechanics, ${ }^{35}$

$$
A^{i d}\left[\left\{\rho_{\alpha}\right\}\right]=k_{B} T \int d \vec{r} \sum_{\alpha=1}^{N} \rho_{\alpha}(\vec{r})\left(\ln \left(\rho_{\alpha}(\vec{r})\right)-1\right),
$$

where $\rho_{\alpha}$ is the density of segment $\alpha, N$ is the number of different types of segments in the system so the summation is conducted over all segments, $k_{B}$ is the Boltzmann constant, and $T$ is the absolute temperature. The de Broglie wavelength is neglected since it does not affect fluid structure.

The hard sphere contribution to the Helmholtz free energy is considered by employing Rosenfeld's fundamental measure theory (FMT), ${ }^{36}$

$$
A^{h s}\left[\left\{\rho_{\alpha}\right\}\right]=k_{B} T \int d \vec{r} \Phi\left[\left\{n_{\alpha}(\vec{r})\right\}\right],
$$

where $\Phi\left[\left\{n_{\alpha}\right\}\right]$ is given by

$$
\begin{aligned}
\Phi\left[\left\{n_{\alpha}(\vec{r})\right\}\right]= & -n_{0} \ln \left(1-n_{3}\right)+\frac{n_{1} n_{2}}{1-n_{3}}+\frac{n_{2}^{3}}{24 \pi\left(1-n_{3}\right)^{2}} \\
& -\frac{\vec{n}_{V 1} \cdot \vec{n}_{V 2}}{1-n_{3}}-\frac{n_{2}\left(\vec{n}_{V 1} \cdot \vec{n}_{V 2}\right)}{8 \pi\left(1-n_{3}\right)^{2}} .
\end{aligned}
$$

$n_{i}(i=0,1,2,3, V 1, V 2)$ are FMT weighted densities given by

$$
\begin{aligned}
n_{i}(\vec{r}) & =\sum_{\alpha=1}^{N} n_{i, \alpha}(\vec{r}) \\
& =\sum_{\alpha=1}^{N} \int \rho_{\alpha}^{\text {seg }}\left(\vec{r}_{1}\right) \omega_{\alpha}^{(i)}\left(\vec{r}-\vec{r}_{1}\right) d \vec{r}_{1}, i=0,1,2,3, V 1, V 2,
\end{aligned}
$$

where $\omega_{\alpha}^{(2)}(r)=\delta\left(R_{\alpha}-r\right), \quad \omega_{\alpha}^{(3)}(r)=\Theta\left(R_{\alpha}-r\right), \quad \vec{\omega}_{\alpha}^{(V 2)}(\vec{r})$ $=\frac{\vec{r}}{r} \delta\left(R_{\alpha}-r\right), \omega_{\alpha}^{(0)}(r)=\frac{\omega_{\alpha}^{(2)}(r)}{4 \pi R_{\alpha}^{2}}, \omega_{\alpha}^{(1)}(r)=\frac{\omega_{\alpha}^{(2)}(r)}{4 \pi R_{\alpha}}$, and $\vec{\omega}_{\alpha}^{(V 1)}(\vec{r})$ $=\frac{\omega_{\alpha}^{(V 2)}(\vec{r})}{4 \pi R_{\alpha}} \cdot \delta\left(R_{\alpha}-r\right)$ is the Dirac delta function and $\Theta\left(R_{\alpha}-r\right)$ is the Heaviside step function, where $R_{\alpha}$ is the radius of segment $\alpha$.

The Helmholtz free energy resulting from long-range attraction is considered by neglecting the detailed correlation between segments (mean-field approximation), ${ }^{37}$

$$
\begin{aligned}
A^{\text {att }}\left[\left\{\rho_{\alpha}\right\}\right]= & \frac{1}{2} k_{B} T \sum_{\alpha=1}^{N} \sum_{\beta=1}^{N} \int_{\left|\overrightarrow{r_{2}}-\overrightarrow{r_{1}}\right|>\sigma_{\alpha \beta}} d \overrightarrow{r_{1}} d \overrightarrow{r_{2}} u_{\alpha \beta}\left(\left|\overrightarrow{r_{2}}-\overrightarrow{r_{1}}\right|\right) \\
& \times \rho_{\alpha}\left(\overrightarrow{r_{1}}\right) \rho_{\beta}\left(\overrightarrow{r_{2}}\right),
\end{aligned}
$$

where $u_{\alpha \beta}$ is given in Eq. (1).

The Helmholtz free energy due to association was derived from Wertheim's theory. ${ }^{38-41}$ It can be written as ${ }^{34,42,43}$

$$
\begin{aligned}
A^{a s \mathrm{soc}}\left[\left\{\rho_{\alpha}\right\}\right]= & k_{B} T \int d \overrightarrow{r_{1}} \sum_{\alpha=1}^{N} \rho_{\alpha}\left(\overrightarrow{r_{1}}\right) \\
& \times \sum_{A \in \Gamma^{(\alpha)}}\left(\ln X_{A}^{\alpha}\left(\overrightarrow{r_{1}}\right)-\frac{X_{A}^{\alpha}\left(\overrightarrow{r_{1}}\right)}{2}+\frac{1}{2}\right),
\end{aligned}
$$


where $X_{A}^{\alpha}$ is the fraction of association sites $A$ on segments $\alpha$ which are not bonded. This fraction is given by $X_{A}^{\alpha}\left(\overrightarrow{r_{1}}\right)=\frac{1}{1+\sum_{\alpha^{\prime}=1}^{N} \int d \overrightarrow{r_{2}} \rho_{\alpha^{\prime}}\left(\overrightarrow{r_{2}}\right) \sum_{B \in \Gamma\left(\alpha^{\prime}\right)} X_{B}^{\alpha^{\prime}\left(\overrightarrow{r_{2}}\right) \Delta \alpha \alpha^{\prime}\left(\vec{r}_{1}, \overrightarrow{r_{2}}\right)}}$, where $\alpha^{\prime}$ represents the segments which can associate or form hydrogen bond with segments $\alpha, \Gamma^{\left(\alpha^{\prime}\right)}$ denotes all association sites on segment $\alpha^{\prime}$, and $\Delta^{\alpha \alpha^{\prime}}\left(\overrightarrow{r_{1}}, \overrightarrow{r_{2}}\right)$ is given by

$$
\Delta^{\alpha \alpha^{\prime}}\left(\overrightarrow{r_{1}}, \overrightarrow{r_{2}}\right)=\kappa\left[\exp \left(\beta \varepsilon_{\text {assoc }}\right)-1\right] y^{\alpha \alpha^{\prime}}\left(\overrightarrow{r_{1}}, \overrightarrow{r_{2}}\right),
$$

where $\kappa$ is a bonding geometric constant, $\beta$ is the inverse temperature $\frac{1}{k_{B} T}, \varepsilon_{a s s o c}$ is the association energy, and $y^{\alpha \alpha^{\prime}}\left(\overrightarrow{r_{1}}, \overrightarrow{r_{2}}\right)$ is the inhomogeneous cavity correlation function of the reference hard sphere fluid. Assuming that the potential of mean force is pairwise additive and using a weighted density approximation, this function can be approximated as ${ }^{26,28}$

$$
y^{\alpha \alpha^{\prime}}\left(\overrightarrow{r_{1}}, \overrightarrow{r_{2}}\right)=\sqrt{y_{\text {contact }}^{\alpha \alpha^{\prime}}\left[\left\{\bar{\rho}_{\alpha}\left(\vec{r}_{1}\right)\right\}\right] y_{\text {contact }}^{\alpha \alpha^{\prime}}\left[\left\{\bar{\rho}_{\alpha}\left(\overrightarrow{r_{2}}\right)\right\}\right]},
$$

where $y_{\text {contact }}^{\alpha \alpha^{\prime}}\left[\left\{\bar{\rho}_{\alpha}(\vec{r})\right\}\right]$ is the bulk cavity correlation function at contact evaluated at weighted densities $\left\{\bar{\rho}_{\alpha}\right\}$. In this work, $y_{\text {contact }}^{\alpha \alpha^{\prime}}\left[\left\{\bar{\rho}_{\alpha}(\vec{r})\right\}\right]$ is given by ${ }^{28}$

$$
\begin{aligned}
y_{\text {contact }}^{\alpha \alpha^{\prime}}\left[\left\{\bar{\rho}_{\alpha}\left(\vec{r}_{1}\right)\right\}\right]= & \frac{1}{1-\overline{\varsigma_{3}}}+\frac{3 \sigma_{\alpha} \sigma_{\alpha^{\prime}}}{\sigma_{\alpha}+\sigma_{\alpha^{\prime}}} \frac{\overline{\varsigma_{2}}}{\left(1-\overline{\varsigma_{3}}\right)^{2}} \\
& +2\left(\frac{\sigma_{\alpha} \sigma_{\alpha^{\prime}}}{\sigma_{\alpha}+\sigma_{\alpha^{\prime}}}\right)^{2} \frac{\left(\overline{\varsigma_{2}}\right)^{2}}{\left(1-\overline{\varsigma_{3}}\right)^{3}},
\end{aligned}
$$

where $\overline{\varsigma_{i}}$ is given by

$$
\overline{\varsigma_{i}}=\frac{\pi}{6} \sum_{\alpha=1}^{N} \bar{\rho}_{\alpha}\left(\overrightarrow{r_{1}}\right) \sigma_{\alpha}^{i}, \quad i=0,1,2,3 .
$$

The weighted densities in the above equations are

$$
\bar{\rho}_{\alpha}\left(\overrightarrow{r_{1}}\right)=\frac{3}{4 \pi \sigma_{\alpha}{ }^{3}} \int_{\left|\overrightarrow{r^{\prime}}-\vec{r}_{1}\right|<\sigma_{\alpha}} d \vec{r}^{\prime} \rho_{\alpha}\left(\vec{r}^{\prime}\right) .
$$

By taking the limit $\varepsilon_{\text {assoc }} \rightarrow \infty$, we reach complete association and chain formation. The free energy due to chain formation $A^{\text {chain }}$ can be directly derived from $A^{\text {assoc }}$ (see the original publication $^{29}$ for more details).

As of now, the total Helmholtz free energy is obtained,

$$
A=A^{i d}+A^{h s}+A^{a t t}+A^{a s s o c}+A^{\text {chain }} .
$$

The grand potential of a grand canonical ensemble, where volume $V$, temperature $T$, and chemical potential $\mu$ of each species are fixed, can be written as

$$
\Omega\left[\left\{\rho_{\alpha}\right\}\right]=A\left[\left\{\rho_{\alpha}\right\}\right]-\sum_{\alpha=1}^{N} \int d \vec{r}^{\prime} \rho_{\alpha}\left(\vec{r}^{\prime}\right)\left(\mu_{\alpha}-V_{\alpha}{ }^{e x t}\left(\vec{r}^{\prime}\right)\right),
$$

where $\mu_{\alpha}$ is the chemical potential of segments $\alpha$, and $V_{\alpha}{ }^{\text {ext }}$ is the external potential acting on segments $\alpha$.

By minimizing the grand potential with respect to densities, the equilibrium density profiles $\rho_{\alpha}^{\text {equilibrium }}(\vec{r})$ can be obtained,

$$
\left.\frac{\delta \Omega\left[\left\{\rho_{\alpha}\right\}\right]}{\delta \rho_{\alpha}(\vec{r})}\right|_{\rho_{\alpha} \text { equilibrium }}=0, \quad \forall \alpha \in\{1,2, \ldots, N\} .
$$

Combining Eqs. (17) and (18) gives

$$
\left.\frac{\delta \beta A\left[\left\{\rho_{\alpha}\right\}\right]}{\delta \rho_{\alpha}(\vec{r})}\right|_{\rho_{\alpha} \text { equilibrium }}=\beta\left(\mu_{\alpha}-V_{\alpha}^{\text {ext }}(\vec{r})\right), \forall \alpha \in\{1,2, \ldots, N\} \text {. }
$$

Solving Eq. (19) requires functional derivatives of the Helmholtz free energy functionals. They are given by

$$
\begin{gathered}
\frac{\delta \beta A^{i d}}{\delta \rho_{\alpha}(\vec{r})},=\ln \rho_{\alpha}(\vec{r}), \\
\frac{\delta \beta A^{h s}}{\delta \rho_{\alpha}(\vec{r})}=\int d \overrightarrow{r_{1}} \frac{\delta \Phi\left[n_{\beta}\left(\overrightarrow{r_{1}}\right)\right]}{\delta \rho_{\alpha}(\vec{r})}, \\
\frac{\delta \beta A^{a t t}}{\delta \rho_{\alpha}(\vec{r})}=\sum_{\gamma=1}^{N} \int_{\left|\vec{r}-\vec{r}_{1}\right|>\sigma_{\alpha \gamma}} d \overrightarrow{r_{1}} \beta u_{\alpha \gamma}^{\text {att }}\left(\left|\vec{r}-\overrightarrow{r_{1}}\right|\right) \rho_{\gamma}\left(\overrightarrow{r_{1}}\right), \\
\frac{\delta \beta A^{a s s o c}}{\delta \rho_{\alpha}(\vec{r})}=\sum_{A \in \Gamma^{(\alpha)}} \ln X_{A}^{\alpha}(\vec{r})-\frac{1}{2} \sum_{\gamma=1}^{N} \sum_{\gamma^{\prime}}^{\left\{\gamma^{\prime}\right\}} \int \rho_{\gamma}\left(\overrightarrow{r_{1}}\right) \\
\times \sum_{A \in \Gamma^{(\gamma)}}\left(1-X_{A}^{\gamma}\left(\overrightarrow{r_{1}}\right)\right) \frac{\delta \ln y_{\text {contact }}^{\gamma \gamma^{\prime}}\left[\left\{\bar{\rho}_{\alpha}\left(\overrightarrow{r_{1}}\right)\right\}\right]}{\delta \rho_{\alpha}(\vec{r})} d \overrightarrow{r_{1}}, \\
\frac{\delta \beta A^{\text {chain }}}{\delta \rho_{\alpha}(\vec{r})}=\sum_{B \in \Gamma^{(\alpha)}} \ln X_{B}^{\alpha}(\vec{r}) \\
-\frac{1}{2} \sum_{\gamma=1}^{N} \sum_{\gamma^{\prime}}^{\left\{\gamma^{\prime}\right\}} \int \rho_{\gamma}\left(\overrightarrow{r_{1}}\right) \frac{\delta \ln y_{\text {contact }}^{\gamma \gamma^{\prime}}\left[\left\{\bar{\rho}_{\alpha}\left(\overrightarrow{r_{1}}\right)\right\}\right]}{\delta \rho_{\alpha}(\vec{r})} d \overrightarrow{r_{1}},
\end{gathered}
$$

where $\Gamma^{(\alpha)}$ denotes the set of association sites on segment $\alpha$ and $\left\{\gamma^{\prime}\right\}$ represents the set of segment that can bond (or associate) with segment $\gamma$ in chain term (or association term). As mentioned above, we have chain formation when association energy is infinity. By $\varepsilon_{a s s o c} \rightarrow \infty$ and $X_{A} \rightarrow 0$, Eq. (23) reduces to Eq. (24). Note that sites $A$ and sites $B$ are conceptually different. Sites $A$ in Eq. (23) denotes association sites that form hydrogen bonds, while sites $B$ in Eq. (24) represents the sites involved in covalent bonds.

After obtaining the functional derivatives, the equations can be solved iteratively for the density profile. Here, a simple Picard iteration is utilized but other techniques such as Newton-Raphson method can also be applied. A computational grid is defined with spacing of $0.05 \sigma$ between density points. To simplify the calculation, we assume that the system is spherically symmetric and the origin of the coordinate system is at the center of the micelle. In other words, the density profile or structure of the micelle is only a function of radial distance $r$ from the center of micelle. The aggregation number (number of surfactant molecules in one micelle) is obtained by integrating the equilibrated density profile over the space. For calculations of interfacial tension isotherm, the interface is assumed planar. The interfacial tension $\gamma$ is the excess grand potential, $\gamma=\frac{\Omega-\Omega_{\text {bulk }}}{A}$, where $\Omega_{\text {bulk }}$ and $\Omega$ are the grand potentials of the bulk phase and of the system, respectively, which are obtained from Eq. (17).

\section{PARAMETER ESTIMATION}

In iSAFT, five physical model parameters, $m, \sigma, \varepsilon, \varepsilon_{a s s o c}$, and $K$, are involved. $m$ represents the number of segments in each molecule, $\sigma$ denotes the hard sphere diameter of a 
segment, $\varepsilon$ represents the depth of Lennard-Jones interaction energy, $\varepsilon_{\text {assoc }}$ is the association energy, and $K$ denotes the bonding volume. The optimum set of these five parameters is regressed such that properties such as vapor pressures and saturated liquid densities calculated from the theory agree with experimental data. In this work, the systems involve water, oil (octane), and surfactant molecules. As mentioned above, water is modeled as one spherical segment with four association sites: two electron donors and two acceptors. Octane is modeled as a chain that consists of 4 tangentially bonded spherical segments, each segment representing two $\mathrm{CH}_{2}$ group. Surfactants, being amphiphilic, are modeled as a chain built of oil-like segments and water-like segments. ${ }^{27,33,44}$

The comparison of vapor pressures and saturated liquid densities between results from iSAFT and experiments is shown in Figure 3. For the segments in the head of surfactants, the water diameter and Lennard-Jones energy are used. Instead of four association sites, each head segment carries two association sites (both are electron donor sites). This model surfactant shares some similarity with the poly (ethylene oxide) alkyl ether (CxEy) surfactants. On one hand, the model surfactant is nonionic and has both hydrophobic and hydrophilic segments. On the other hand, each hydrophilic segment can form hydrogen bond(s) with water, similar to the ethoxy (EO) group in CxEy surfactant. So in Sec. IV, we will compare with experimental data of some CxEy surfactants to qualitatively validate our approach. However, since the parameters of the model surfactant are not determined based on experimental data of CxEy surfactant, only qualitative trend can be discussed. The schematics of the model water, octane, and surfactant molecules are shown in Figure 2. The values of parameters used are given in Table I. Binary interaction parameter between water and octane is fitted to solubility of octane in water, and the final value is 0.07 . For the cross interaction between water and head segments and between octane and tail segments, the binary interaction parameter is 0. For the cross interaction between water and tail segments, and between head segments and octane or tail segments, $\varepsilon / k_{B}$ is set to $100 \mathrm{~K}$, mimicking unfavorable interaction between unlike components.
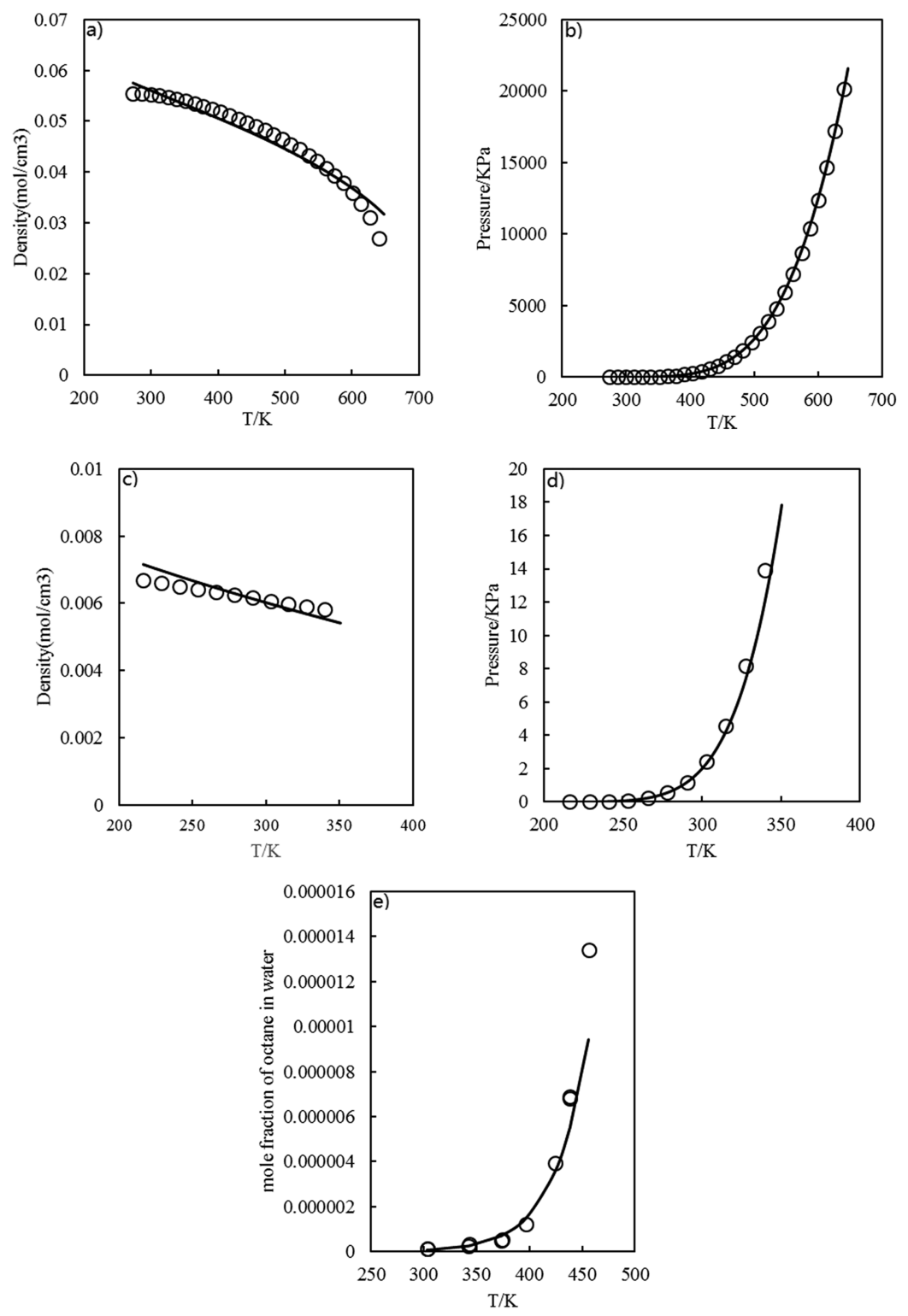

FIG. 3. Liquid density ((a) and (c)) and saturated vapor pressure ((b) and (d)) of water and octane, respectively, from theory (line) and experiments ${ }^{45}$ (circle). Solubility of octane in water (e) from theory (line) and experiments ${ }^{46}$ (circle). 
TABLE I. Summary of parameters.

\begin{tabular}{lccccc}
\hline \hline & $m$ & $\sigma(\AA)$ & $\varepsilon / k_{B}(\mathrm{~K})$ & $\varepsilon_{\text {assoc }} / k_{B}$ & $K / \sigma^{3}$ \\
\hline Water & 1 & 3.0 & 328.6 & 1747.3 & 0.004432 \\
Octane & 4 & 3.6 & 294.05 & & \\
Head segments & $x$ & 3.0 & 328.6 & 1747.3 & 0.004432 \\
Tail segments & $y$ & 3.0 & 294.05 & & \\
\hline
\end{tabular}

\section{RESULTS AND DISCUSSION}

\section{A. Water/surfactant binary mixtures}

\section{Determination of the $\mathrm{CMC}$}

In surfactant systems, the CMC is defined as the minimum concentration of surfactants necessary to form stable micelles. By assuming that the micelle formed is spherically symmetric ${ }^{47}$ the CMC can be determined by iSAFT DFT written in spherical form. Other geometries of the micelles such as rodlike and ellipsoid can also be investigated in iSAFT, which will be the subject of future publications. To obtain the value of the CMC, we compare the grand potentials of the systems with and without the formation of a micelle at the same bulk surfactant concentration. Since volume, temperature, and bulk chemical potentials are fixed, the system with lower grand potential is the stable one. Hence, the CMC can be regarded as the bulk surfactant concentration when the grand potential of system with a micelle is the same as in the system without a micelle.

In Figure 4, we show the grand potential as a function of bulk surfactant mole fraction for $\mathrm{H}_{5} \mathrm{~T}_{4}$ surfactant (5 head segments and 4 tail segments). We can observe that at low surfactant concentration, the system with a micelle in the center has greater grand potential than that without a micelle, which means that the micellar system is not stable and the CMC has not been reached. As we increase the concentration of surfactants in bulk, the grand potential of the micellar system decreases and crosses that without a micelle. At the cross, the micellar phase is as stable as the non-micellar phase;

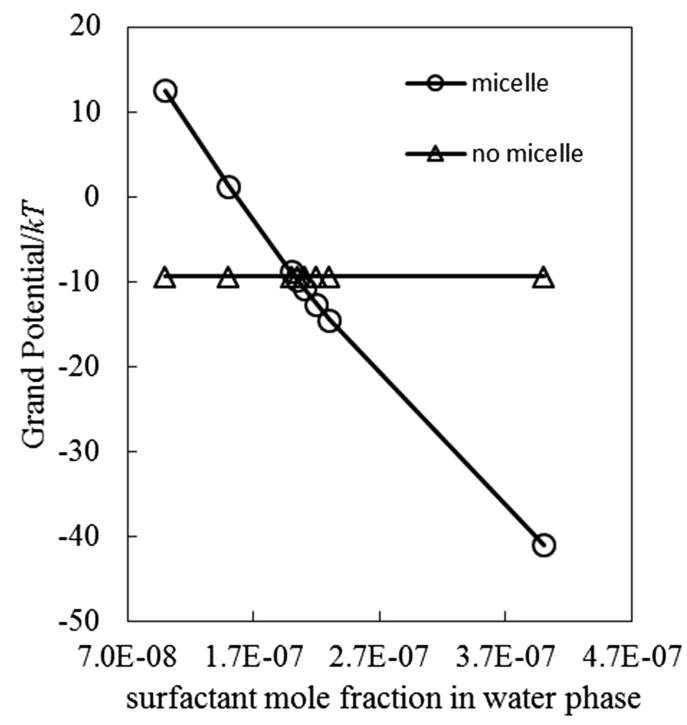

FIG. 4. Comparison of grand potential for the CMC determination.

this is the CMC. If we further increase the bulk concentration of surfactants, the grand potential of micellar phase further decreases, which means that the micellar phase is energetically more favorable and more micelles will form until the bulk surfactant concentration is reduced to the CMC. In our model, the interactions between micelles are not considered. So the system we are studying corresponds to a dilute micellar system.

\section{Micelle structure}

Figure 5(a) shows the reduced number density distribution of tail segments, head segments, and water as a function of radial distance $r$ from the center of a micelle. As we can see, the structure of the micelle formed by $\mathrm{H}_{5} \mathrm{~T}_{4}$ surfactant is similar to the conventional picture of a micelle structure. In the center of the micelle, hydrophobic tail segments aggregate to expel hydrophilic segments and form a hydrophobic core. The iSAFT DFT predicts a depletion of surfactant tail at the
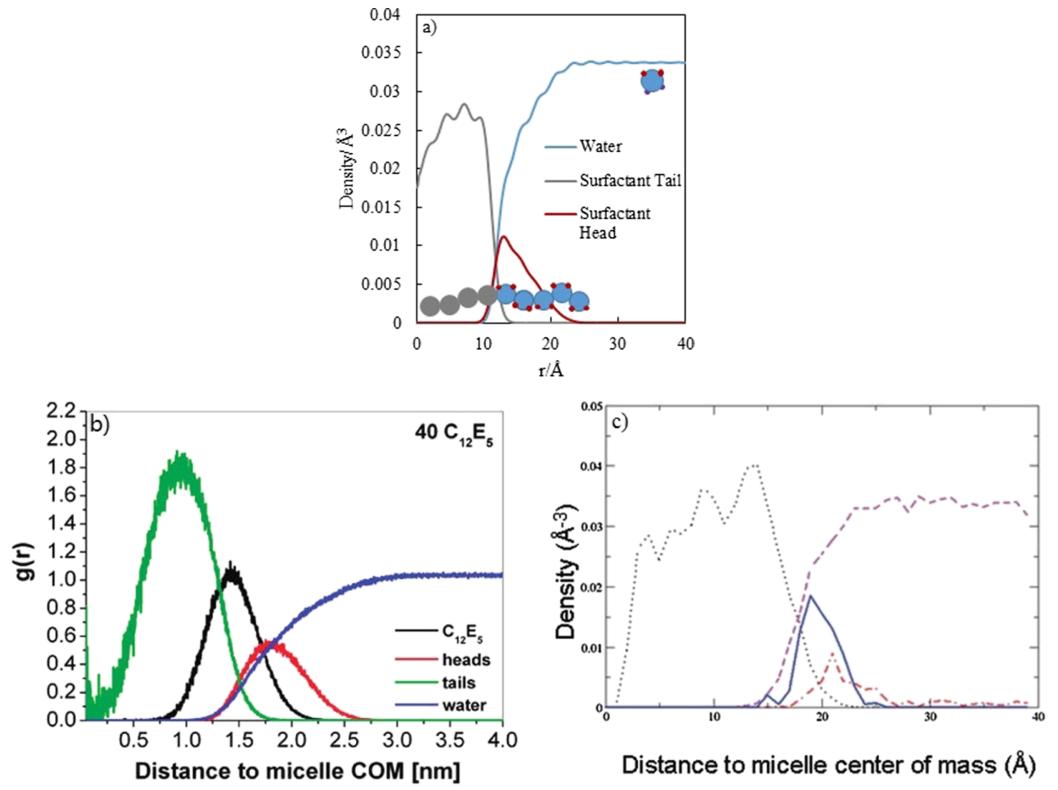

FIG. 5. Structure of a micelle. (a) Density distribution of tail segments, head segments and water molecules of a micelle formed by $\mathrm{H}_{5} \mathrm{~T}_{4}$ surfactants from DFT calculation. (b) The radial distribution functions $g(r)$ of the center of mass $(\mathrm{COM})$ of tails, heads, surfactant molecules, and water molecules for a micelle formed by $40 \mathrm{C}_{12} \mathrm{E}_{5}$ surfactant molecules from molecular dynamics simulation. Reprinted with permission from Velinova et al., Langmuir 27(23), 14071-14077 (2011). Copyright 2011 American Chemical Society. (c) The density distributions of carbons, head group atoms, water molecules and sodium ions for a micelle formed by SDS surfactant molecules from molecular dynamics simulation. Reproduced with permission from Palazzesi et al., Soft Matter 7(19), 9148-9156 (2011). Copyright 2011 The Royal Society of Chemistry. ${ }^{52}$ 
center of micelle, which is also observed in molecular simulations. ${ }^{48,49}$ The average density of hydrophobic segments (T4) in this inner core is approximately equal to the reduced bulk density $\left(\rho \sigma^{3}=0.6815\right)$ of an alkane built of four tail segments at the same temperature. The hydrophilic head segments are exposed to water molecules and fully hydrated. The hydration of the head groups effectively expands the head group helping to stabilize the micelle structure. We can also observe that the radius of the micelle is approximately $8 \sigma$ which corresponds to $24 \AA$; this value is consistent with experimentally measured values ranging from 22 to $24 \AA .50,51$ The water density is essentially zero inside the hydrophobic core of the micelle and increases monotonically to its bulk value. Since the model surfactant shares some similarity with poly(ethylene oxide) alkyl ether (CxEy) surfactant, it is reasonable to compare the micellar structure predicted from our model with that from molecular dynamics simulation ${ }^{48}$ of $\mathrm{CxEy}$, which is reprinted in Figure 5(b). However, the center of mass (COM) of the whole tail moiety, whole head moiety, and the surfactant molecule is used in the MD work for generating the radial distribution functions of the tail, head, and surfactant, respectively, instead of the position of each segment that is the case in iSAFT. The lumping effect introduced by the COM contributes to the discrepancy observed between Figures 5(a) and 5(b). Since the segment based density distributions are not available for CxEy micelles in the literature, we reprinted the density distributions for a micelle formed by SDS surfactants in Figure 5(c). Interestingly, the density of the tails at origin is zero, indicating an unphysical vacuum region in the center of a micelle and we suspect that the micelle simulated has not reached its equilibrated state. Additionally, in MD simulation, the number of surfactants in a micelle is preset, ${ }^{48}$ which may also produce an unphysical structure of the micelle. Nonetheless, overall good agreement can be seen between Figures 5(a) and 5(c). Our iSAFT approach can capture the micelle structure.

\section{Effect of surfactant architecture on the CMC}

The value of CMC depends on the surfactant architecture. ${ }^{16}$ Here, the effect of head size on the CMC is obtained from iSAFT. The size of tail is fixed to 4 tail segments, and the head size ranges from 4 to 7 segments. Experimentally, the $\mathrm{CMC}$ increases monotonically as the head size rises, ${ }^{53}$ same as what we observe from iSAFT (Figure 6(a)). When the head size increases, the hydrophilicity of surfactant goes up, which increases the solubility of surfactant in water. Thus, a higher concentration of surfactant molecules is necessary to form aggregates. The larger than expected change in the CMC with the number of head segments could indicate that the hydrogen bonding interaction is too strong and needs to be revised to show quantitative agreement.

For the effect of tail size on the CMC, the head is fixed to 6 segments and the tail size is changed consecutively from 3 to 6 segments. The result is shown in Figure 6(b). When the size of tail increases, the CMC decreases. This is in qualitative agreement with experimental observation. ${ }^{54}$ The model shows larger dependence of the CMC on tail length than seen experimentally. This might be due to the lack of attraction between water and tail groups in the model.
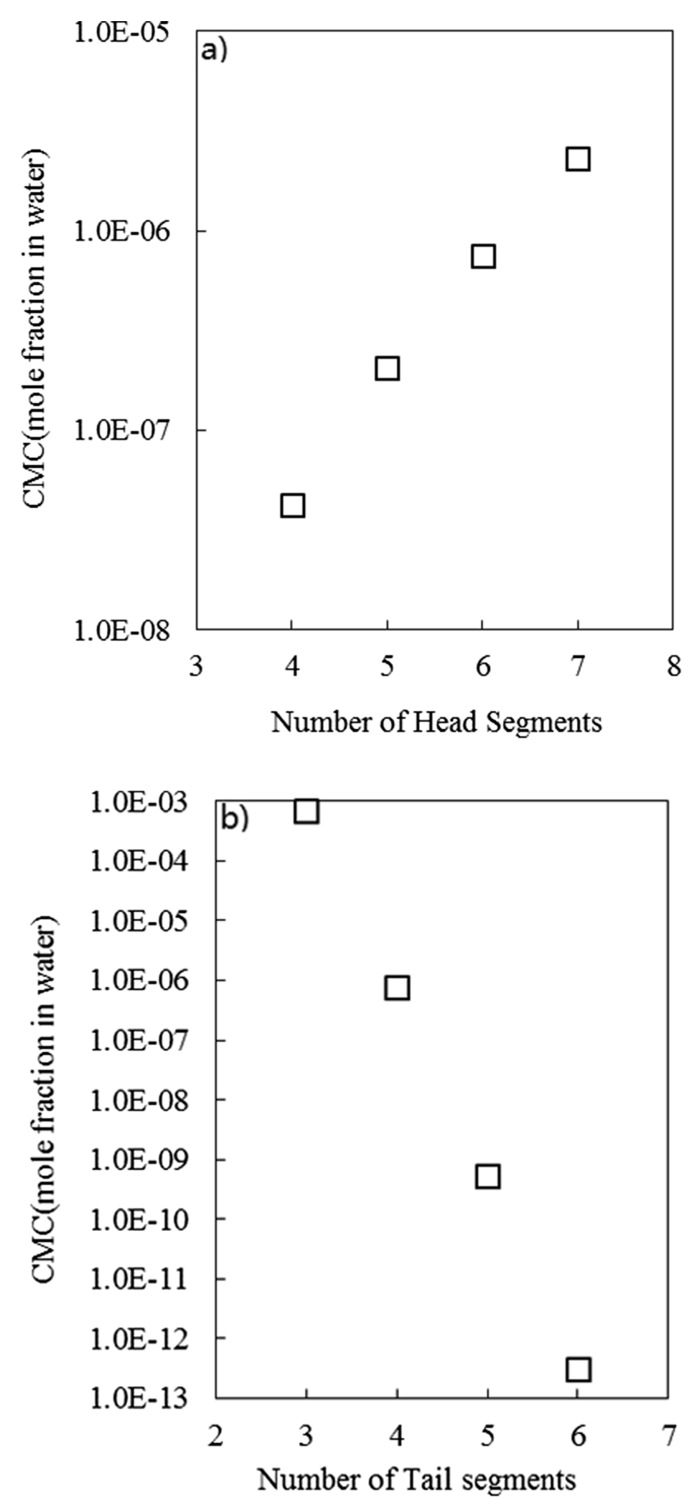

FIG. 6. Effect of surfactant architecture on critical micelle concentration. (a) The effect of head size on the critical micelle concentration. All surfactants have 4 tail segments. (b) The effect of tail size on the critical micelle concentration. All surfactants have 6 head segments.

Experimental results show that the CMC depends much more strongly on the size of tail than that of head. The experimental results ${ }^{53,54}$ showing the $\mathrm{CMC}$ as a function of the size of tail and head are summarized in Table II. We can observe that the CMC remains in the same order of magnitude when the head size is altered from four ethoxy groups to eight ethoxy groups, but it changes orders of magnitude when the tail size is increased from 10 alkyl groups to 14 alkyl groups. This experimental trend can be captured qualitatively by iSAFT. From iSAFT, the CMC rises roughly

TABLE II. Effect of head size and tail size on the CMC from experiment.

\begin{tabular}{lccc}
\hline \hline From Ref. 53 & $\mathrm{C}_{12} \mathrm{E}_{4}$ & $\mathrm{C}_{12} \mathrm{E}_{6}$ & $\mathrm{C}_{12} \mathrm{E}_{8}$ \\
\hline $\mathrm{CMC}\left(\mathrm{mol} / \mathrm{cm}^{3}\right)$ at $30{ }^{\circ} \mathrm{C}$ & $\begin{array}{c}5.3 \times 10^{-8} \\
\mathrm{C}_{10} \mathrm{E}_{8}\end{array}$ & $\begin{array}{c}6.8 \times 10^{-8} \\
\mathrm{C}_{12} \mathrm{E}_{8}\end{array}$ & $\begin{array}{c}7.6 \times 10^{-8} \\
\mathrm{C}_{14} \mathrm{E}_{8}\end{array}$ \\
From Ref. 54 & $9.3 \times 10^{-4}$ & $6.9 \times 10^{-5}$ & $8.0 \times 10^{-6}$ \\
\hline $\mathrm{CMC}(\mathrm{mol} / \mathrm{l})$ at $30^{\circ} \mathrm{C}$ & &
\end{tabular}


by 4 times when the head size is increased by one ethoxy group, but it decreases roughly by 40 times when the tail size is increased by one $\mathrm{CH}_{2}$ group (one tail segment represents 2 $\mathrm{CH}_{2}$ groups). Hence, the $\mathrm{CMC}$ depends much more strongly on the size of surfactant tail, which is consistent with experimental observation.

\section{Effect of surfactant structure on aggregation number}

Another physical quantity associated with surfactant systems is the aggregation number. It describes the number of surfactant molecules present in a micelle. This quantity is relatively hard to measure experimentally, involving the use of static light scattering, ${ }^{55}$ fluorescence quenching methods, ${ }^{56}$ etc. And the aggregation number measured experimentally is the mean aggregation number but the micelles are not truly monodisperse; there is continual interchange between molecules in the bulk phase and the micelles. In iSAFT, the mean aggregation number of a micelle can be calculated from
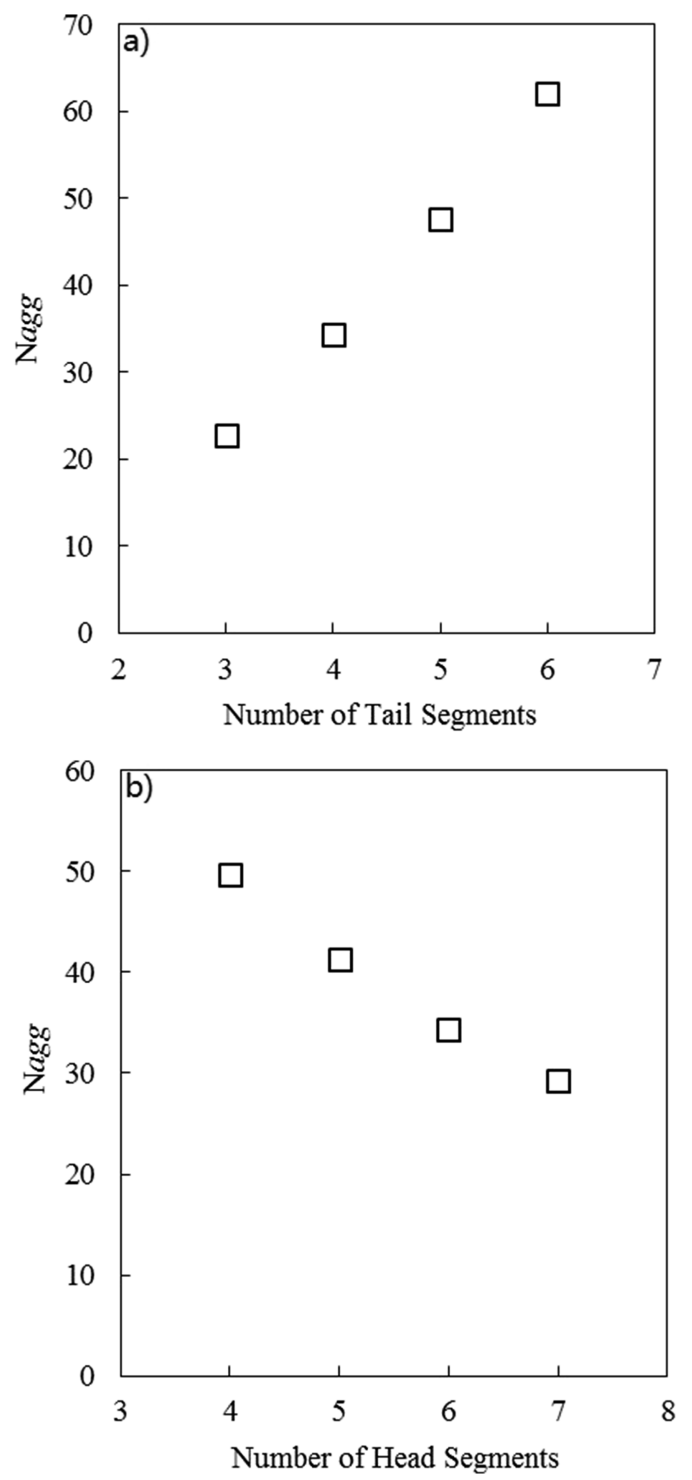

FIG. 7. Effect of surfactant structure on aggregation number. (a) The effect of tail size on the aggregation number. All surfactants have 6 head segments. (b) The effect of head size on the aggregation number. All surfactants have 4 tail segments. the density profile, and the results are shown in Figure 7. From the figure, we can see that the aggregation number is in the range of 30-70; this is consistent with experimental values in the range of ionic and nonionic surfactant molecules. ${ }^{56}$ It is observed that when the size of tail increases, the number of surfactant molecules present in the micelle also rises, but when the size of head increases, the aggregation number decreases. In other words, when the hydrophilicity of surfactant increases (either by decreasing the tail size or increasing the head size), the aggregation number would decrease. This observation can be explained from the point of view of surfactant packing in the micelle. ${ }^{57}$ The conical shape of the hydrated surfactant determines the size of the micelle. Larger tail or smaller head would result in a favorable shape contributing to greater aggregation number.

\section{Effect of surfactant structure on interfacial tension}

Due to its amphiphilic nature, surfactant molecules spontaneously partition to the interface and lower the interfacial tension. This is why surfactants find great application
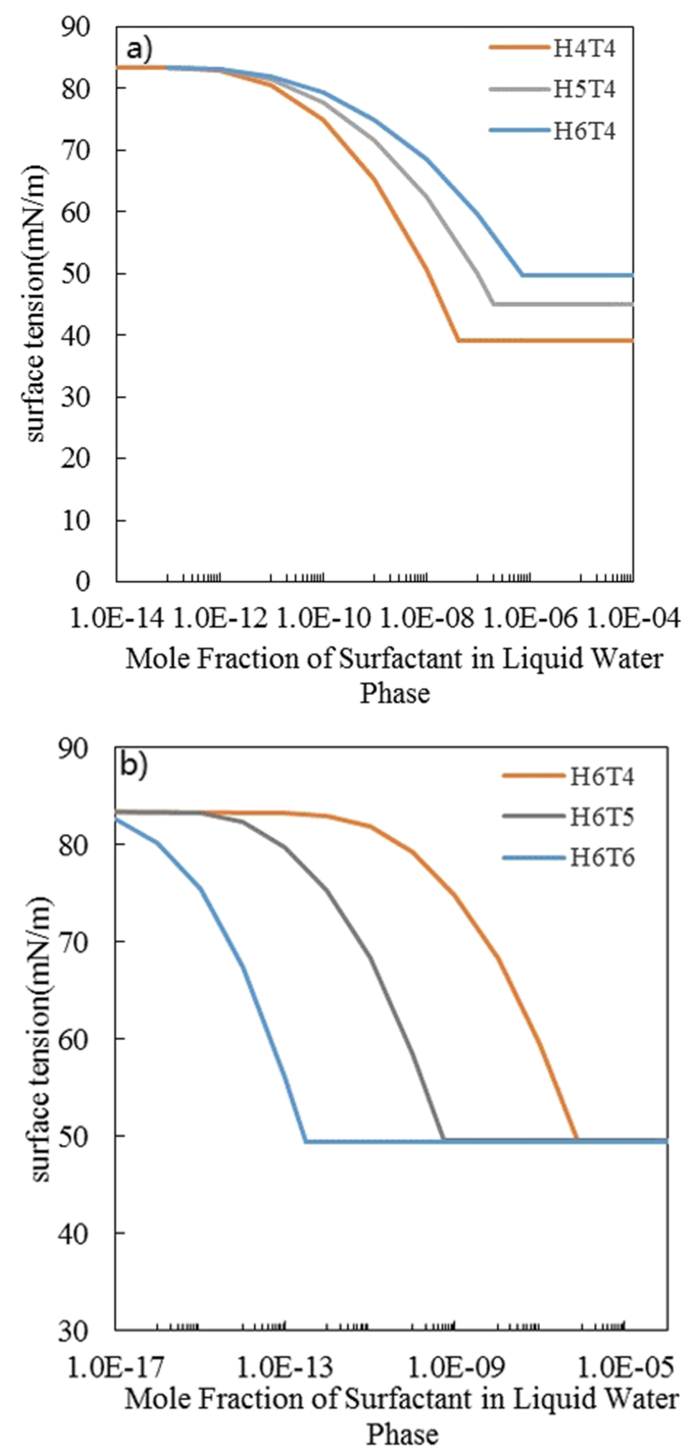

FIG. 8. Effect of surfactant architecture on surface tension from iSAFT calculation. (a) Effect of head size. (b) Effect of tail size. 

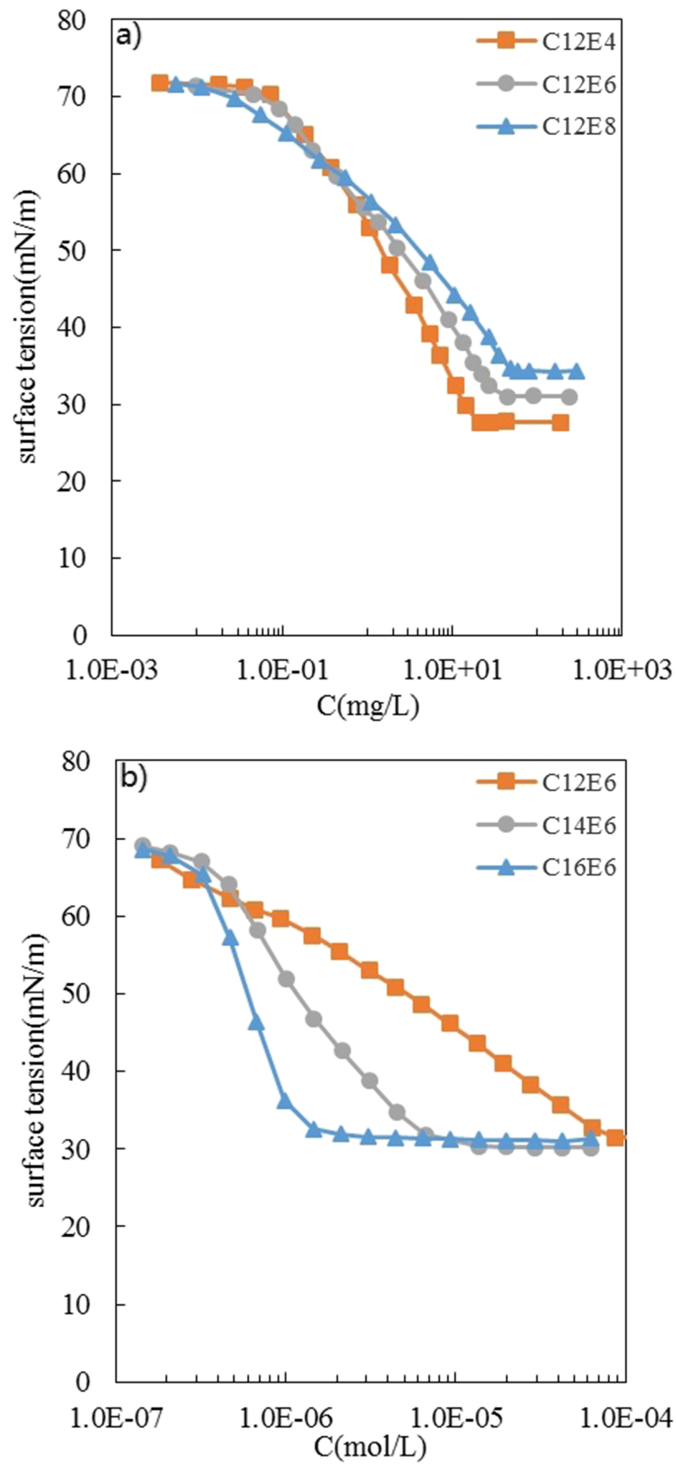

FIG. 9. Experimental surface tension isotherm. (a) Reprinted with permission from Lin et al., Langmuir 15(13), 4370-4376 (1999). Copyright 1999 American Chemical Society. (b) Reprinted with permission from Zhmud et al., Langmuir 16(6), 2557-2565 (2000). Copyright 2000 American Chemical Society.

in the field of enhanced oil recovery, personal care products, etc. ${ }^{1,2}$ For these applications, it is helpful to have some insights into the effect of surfactant structure on interfacial or surface tension. Previous work with iSAFT ${ }^{27}$ did not consider micelle formation and specific association sites. In this work, we take both micellization and association into account, and obtain the interfacial tension isotherm for each specific surfactant.

Here we investigate the binary mixtures consisting of water and surfactant. The surfactants of interest are $\mathrm{H}_{4} \mathrm{~T}_{4}$, $\mathrm{H}_{5} \mathrm{~T}_{4}, \mathrm{H}_{6} \mathrm{~T}_{4}, \mathrm{H}_{6} \mathrm{~T}_{5}$, and $\mathrm{H}_{6} \mathrm{~T}_{6}$. The CMCs were obtained by conducting iSAFT calculations in spherical coordinates at constant temperature ( $298 \mathrm{~K}$ ) and pressure (1 bar). The interfacial tensions $\gamma$ are obtained from iSAFT by calculating the vapor-liquid interfacial profile of a planar interface at constant temperature and over a range of surfactant concentrations with $\gamma=\frac{\Omega-\Omega_{\text {bulk }}}{A}$. Here, the assumptions we made are as follows: (1) the surface is planar and symmetric in the $x-y$ plane; (2)

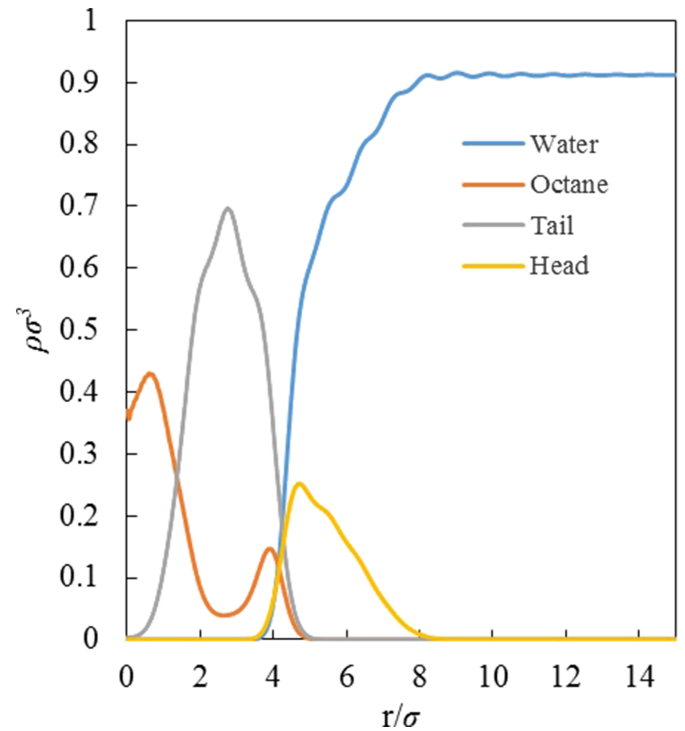

FIG. 10. Structure of swollen micelle formed by H5T4/octane/water at $298 \mathrm{~K}$ and 1 bar.

the CMCs are not changed when the pressure is changed from $1 \mathrm{bar}$ to vapor/liquid coexistence condition at $298 \mathrm{~K}$ since such a small change in pressure has little effect on liquid phase properties.

The iSAFT results, shown in Figure 8, are in very good qualitative agreement with experimental results given by Lin et al. ${ }^{58}$ and Zhmud et al. ${ }^{59}$ which are reprinted in Figure 9. Some observations can be made. The interfacial tension is constant for surfactant concentrations above the CMC since additional surfactant partitions to micelles instead of the vaporliquid interface. We also observe that a surfactant with smaller head or larger tail is more efficient in lowering the interfacial tension when the concentration of surfactant is below its CMC. Thus a more hydrophobic or less hydrophilic surfactant partitions more readily to the interface and has a lower CMC. From Fig. 8(b), it can be observed that each increase in surfactant carbon number shifts the interfacial tension curve by a constant factor. This is known as the Traube's rule ${ }^{60}$ which describes the relationship between hydrocarbon chain length and surfactant activity. A surfactant with a smaller head can reach lower interfacial tensions at concentrations above the CMC, which is in qualitative agreement with experiment. ${ }^{61}$ Interestingly, the interfacial tension above the CMC is independent of tail length. So the effectiveness of a surfactant in lowering the interfacial tension is more affected by the head of the surfactant.

\section{B. Water/octane/surfactant ternary mixtures}

\section{Critical micelle concentration and swollen micelle}

The results of water/surfactant binary mixtures were discussed, but what will happen to the micelles when we introduce oil into the system? iSAFT predicts the tendency for oil to be solubilized in the center of a micelle, a swollen micelle.

The CMC for a ternary mixture is determined similar to that for a binary mixture. A series of calculations are done at $25{ }^{\circ} \mathrm{C}$ and 1 bar with different concentrations of surfactant in the aqueous phase. The CMC is determined based on the grand potential of the system with or without a swollen 

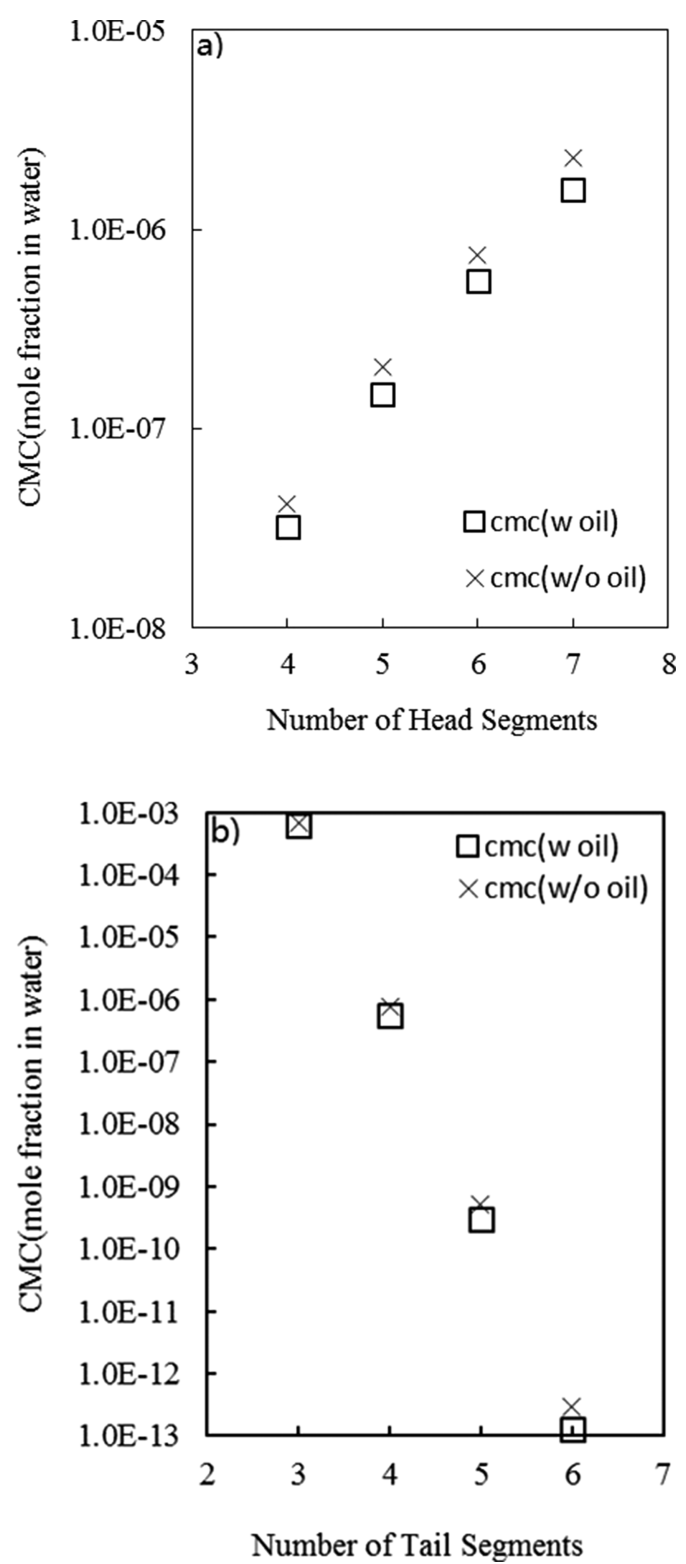

FIG. 11. Comparison of the CMC values of surfactant/water systems and surfactant/octane/water systems. (a) The effect of head size on the critical micelle concentration. All surfactants have 4 tail segments. Squares are CMCs with oil present and crosses are CMCs without oil. (b) The effect of tail size on the critical micelle concentration. All surfactants have 6 head segments. Squares are CMCs with oil present and crosses are CMCs without oil.

micelle formed. The structure of a swollen micelle is shown in Figure 10. It is observed that octane molecules accumulate in the center of the micelle and surfactant helps to decrease the contact between octane and water, hence lowering the free energy of the system.

The CMCs of octane/water/surfactant ternary mixtures are given in Figure 11. The CMCs follow the same trend as those of water/surfactant binary mixtures. The presence of oil slightly decreases the CMC values because octane helps stabilize the micelle ${ }^{61}$ in the same way as increasing the surfactant tail length reduces the CMC.

\section{Reverse swollen micelle}

The shape of surfactant molecules determines the structure of surfactant aggregates. When a surfactant molecule
TABLE III. iSAFT parameters for surfactant that forms reverse micelle.

\begin{tabular}{lccccc}
\hline \hline & $m$ & $\sigma(\AA)$ & $\varepsilon / k_{B}(\mathrm{~K})$ & $\varepsilon_{\text {assoc }} / k_{B}$ & $K / \sigma^{3}$ \\
\hline Head segments & $x$ & 3.0 & 328.6 & 1247.3 & 0.004432 \\
Tail segments & $y$ & 3.6 & 294.05 & & \\
\hline \hline
\end{tabular}

carries a large head and small tail, its conical shape would favor the formation of micelle in water. However, if the molecule carries a large tail and small head, the formation of micelle in water would not be that favorable since it is difficult for the molecules to fit. Instead of forming micelles in water, formation of reverse micelles in oil may be more favorable. With this simple idea, researchers ${ }^{57,62}$ proposed the surfactant packing parameter to describe the favorable types of micelle to be formed by different surfactants.

By employing the iSAFT potential parameters listed in Table I, reverse swollen micelle cannot be obtained since the shape of surfactant molecule described by those parameters does not favor the reverse micelle formation. The shape of surfactant molecule may be altered by changing conditions such as temperature, ${ }^{62}$ but the study of temperature effect is beyond the scope of this paper and will be considered in the following papers. Instead of altering conditions, we simply choose values of parameters more favorable to form a reverse micelle. The set of parameters are given in Table III.

From the table, we can see that the diameter of tail segments is increased and the association energy between surfactant head and water is decreased. As a result, the surfactant molecules have a larger tail and smaller head, which favors the formation of reverse micelle. Following the same procedure described above, the calculated reverse swollen micelle structure is shown in Figure 12. When the system free energy is minimized, it is predicted that water molecules accumulate in the center of the reverse micelle.

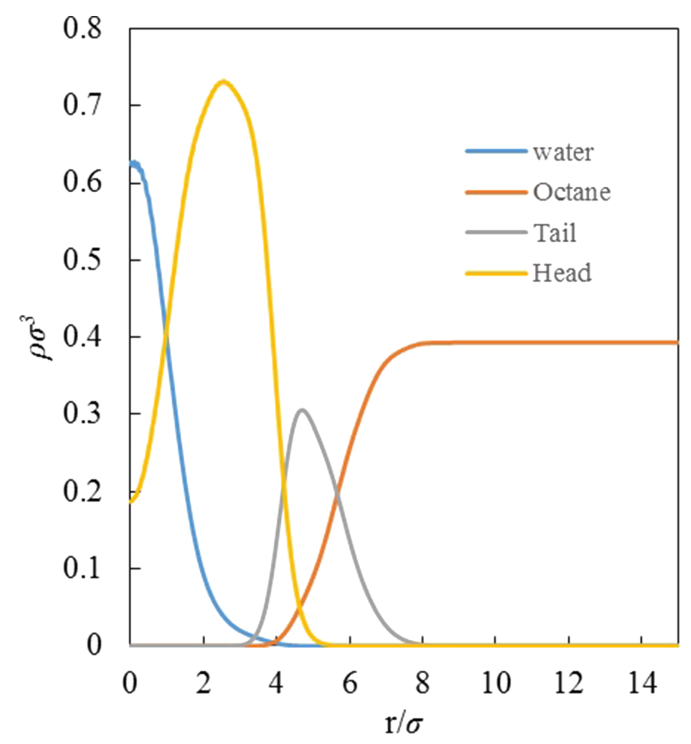

FIG. 12. Structure of reverse micelle formed by H5T4/water/octane using the parameters listed in Table III. 


\section{CONCLUSION}

In this work, iSAFT classical density functional theory was extended to study the effects of surfactant architecture on critical micelle concentration, aggregation number, and interfacial tension. Our model includes explicit hydration of the head groups that has been shown important to the size of the micelle $e^{63}$ and the phase behavior of surfactant systems. ${ }^{64}$ The model correctly predicted that CMC increases with an increase of head size and decreases with an increase of tail size. An opposite trend was observed for the dependence of aggregation number on the structure of surfactant molecules. Additionally, the interfacial tension isotherms of vapor/liquid interfaces for the model surfactants were calculated. It was shown that the interfacial tension at CMC increases with an increase in head size but does not depend on the tail size. Traube's rule which describes the dependence of surfactant's surface activity on the tail size was also captured in the iSAFT model. All of these observations are in qualitative agreement with experimental results. Furthermore, the formation of swollen micelle and reverse swollen micelle is also described by this model. To our knowledge, this is the first time classical density functional theory, particularly with explicit hydration of the head groups, has been applied to such systems.

Since the parameters of surfactant molecules have not been fit to the experimental data, a quantitative description of the thermodynamic properties of CxEy surfactant systems has not been reached. Developing a surfactant model to better fit experimental data will be the subject of future publications. Additionally, since the iSAFT used in this work is based on Wertheim's first order thermodynamic perturbation theory(TPT1), the surfactant molecule is flexible and no bond rigidity is considered which can be included by a TPT2 approach. ${ }^{44}$ Free energy contributions due to partial charges on surfactant molecules ${ }^{12}$ may also be developed and included in the iSAFT framework for a more realistic description of the systems containing ionic surfactants. In the future, investigations on the effects of external conditions such as temperature and pressure on the formation of the micelle and the formation of microemulsions will be made. Further, the method of moments will be used to calculate the phase inversion temperature and to help isolate conditions for middle phase microemulsion formation. The ultimate goal is to apply iSAFT in the molecular design of surfactant formations for enhanced oil recovery and other applications.

\section{SUPPLEMENTARY MATERIAL}

See supplementary material for the numerical integrals in 1-D spherical coordinate used for conducting the iSAFT calculations.

\section{ACKNOWLEDGMENTS}

The authors gratefully acknowledge the Rice University Consortium for Processes in Porous Media and the Robert A. Welch Foundation (Grant No. C-1241) for financial support.
${ }^{1}$ G. J. Hirasaki, C. A. Miller, and M. Puerto, "Recent advances in surfactant EOR," SPE Annual Technical Conference and Exhibition (Society of Petroleum Engineers, 2008)

${ }^{2}$ S. H. Im, Y. H. Jeong, and J. J. Ryoo, "Simultaneous analysis of anionic, amphoteric, nonionic and cationic surfactant mixtures in shampoo and hair conditioner by RP-HPLC/ELSD and LC/MS," Anal. Chim. Acta 619(1), 129-136 (2008).

${ }^{3}$ P. A. Winsor, Solvent Properties of Amphiphilic Compounds (Butterworths Scientific Publications, 1954).

${ }^{4}$ J. G. E. M. Fraaije, K. Tandon, S. Jain, J.-W. Handgraaf, and M. Buijse, "Method of moments for computational microemulsion analysis and prediction in tertiary oil recovery," Langmuir 29(7), 2136-2151 (2013).

${ }^{5}$ R. D. Groot and P. B. Warren, "Dissipative particle dynamics: Bridging the gap between atomistic and mesoscopic simulation," J. Chem. Phys. 107(11), 4423 (1997).

${ }^{6}$ J. M. Vanegas, A. Torres-Sánchez, and M. Arroyo, "Importance of force decomposition for local stress calculations in biomembrane molecular simulations," J. Chem. Theory Comput. 10(2), 691-702 (2014).

${ }^{7}$ A. L. Frischknecht and L. J. Douglas Frink, "Comparison of density functional theory and simulation of fluid bilayers," Phys. Rev. E 72(4), 041924 (2005).

${ }^{8}$ E. C. Voutsas, M. V. Flores, N. Spiliotis, G. Bell, P. J. Halling, and D. P. Tassios, "Prediction of critical micelle concentrations of nonionic surfactants in aqueous and nonaqueous solvents with UNIFAC," Ind. Eng. Chem. Res. 40(10), 2362-2366 (2001).

${ }^{9}$ X.-S. Li, J.-F. Lu, Y.-G. Li, and J.-C. Liu, "Studies on UNIQUAC and SAFT equations for nonionic surfactant solutions," Fluid Phase Equilib. 153(2), 215-229 (1998).

${ }^{10}$ Y. Moroi, "Mass action model of micelle formation: Its application to sodium dodecyl sulfate solution,” J. Colloid Interface Sci. 122(2), 308-314 (1988).

${ }^{11}$ R. Nagarajan and E. Ruckenstein, "Theory of surfactant self-assembly: A predictive molecular thermodynamic approach," Langmuir 7(12), 2934-2969 (1991).

${ }^{12}$ S. Puvvada and D. Blankschtein, "Molecular-thermodynamic approach to predict micellization, phase behavior and phase separation of micellar solutions. I. Application to nonionic surfactants," J. Chem. Phys. 92(6), 3710-3724 (1990).

${ }^{13}$ S. Puvvada and D. Blankschtein, "Thermodynamic description of micellization, phase behavior, and phase separation of aqueous solutions of surfactant mixtures," J. Phys. Chem. 96(13), 5567-5579 (1992).

${ }^{14}$ S. Puvvada and D. Blankschtein, "Theoretical and experimental investigations of micellar properties of aqueous solutions containing binary mixtures of nonionic surfactants," J. Phys. Chem. 96(13), 5579-5592 (1992).

${ }^{15}$ B. Lukanov and A. Firoozabadi, "Molecular thermodynamic modeling of reverse micelles and water-in-oil microemulsions," Langmuir 32(13), 3100-3109 (2016).

${ }^{16} \mathrm{H}$. B. Klevens, "Structure and aggregation in dilate solution of surface active agents," J. Am. Oil Chem. Soc. 30(2), 74-80 (1953).

${ }^{17}$ C. Lian, L. Wang, X. Chen, X. Han, S. Zhao, H. Liu, and Y. Hu, "Modeling swelling behavior of thermoresponsive polymer brush with lattice density functional theory," Langmuir 30(14), 4040-4048 (2014).

${ }^{18}$ C. Lian, X. Chen, S. Zhao, W. Lv, X. Han, H. Wang, and H. Liu, "Substrate effect on the phase behavior of polymer brushes with lattice density functional theory," Macromol. Theory Simul. 23(9), 575-582 (2014).

${ }^{19}$ K. Gong and W. G. Chapman, "Solvent response of mixed polymer brushes," J. Chem. Phys. 135(21), 214901 (2011).

${ }^{20} \mathrm{~K}$. Gong, B. D. Marshall, and W. G. Chapman, "Response behavior of diblock copolymer brushes in explicit solvent," J. Chem. Phys. 137(15), 154904 (2012).

${ }^{21}$ K. Gong, B. D. Marshall, and W. G. Chapman, "Modeling lower critical solution temperature behavior of associating polymer brushes with classical density functional theory," J. Chem. Phys. 139(9), 094904 (2013).

${ }^{22}$ Z. Feng and W. G. Chapman, "Revisited block copolymer/nanoparticle composites: Extension of interfacial statistical associating fluid theory," Macromolecules 45(16), 6658-6668 (2012).

${ }^{23} \mathrm{D}$. Cao and J. Wu, "Microstructure of block copolymers near selective surfaces: Theoretical predictions and configurational-bias Monte Carlo simulation," Macromolecules 38(3), 971-978 (2005).

${ }^{24} \mathrm{D}$. Cao and J. Wu, "Surface-induced phase transitions in ultrathin films of block copolymers," J. Chem. Phys. 122(19), 194703 (2005).

${ }^{25}$ D. Ballal and W. G. Chapman, "Hydrophobic and hydrophilic interactions in aqueous mixtures of alcohols at a hydrophobic surface," J. Chem. Phys. 139(11), 114706 (2013). 
${ }^{26}$ B. D. Marshall, K. R. Cox, and W. G. Chapman, "A classical density functional theory study of the neat n-alkane/water interface," J. Phys. Chem. C 116(33), 17641-17649 (2012).

${ }^{27}$ C. P. Emborsky, K. R. Cox, and W. G. Chapman, "Exploring parameter space effects on structure-property relationships of surfactants at liquidliquid interfaces," J. Chem. Phys. 135(8), 084708 (2011).

${ }^{28}$ S. Tripathi and W. G. Chapman, "Microstructure of inhomogeneous polyatomic mixtures from a density functional formalism for atomic mixtures," J. Chem. Phys. 122(9), 094506 (2005).

${ }^{29}$ S. Jain, A. Dominik, and W. G. Chapman, "Modified interfacial statistical associating fluid theory: A perturbation density functional theory for inhomogeneous complex fluids," J. Chem. Phys. 127(24), 244904 (2007).

${ }^{30}$ W. G. Chapman, K. E. Gubbins, G. Jackson, and M. Radosz, "New reference equation of state for associating liquids," Ind. Eng. Chem. Res. 29(8), 1709-1721 (1990).

${ }^{31}$ C. J. Segura and W. G. Chapman, "Associating fluids with four bonding sites against solid surfaces: Monte Carlo simulations," Mol. Phys. 86(3), 415-442 (1995).

${ }^{32}$ S. Tripathi and W. G. Chapman, "Density-functional theory for polar fluids at functionalized surfaces. I. Fluid-wall association," J. Chem. Phys. 119(23), 12611-12620 (2003).

${ }^{33}$ M. M. Telo da Gama and K. E. Gubbins, "Adsorption and orientation of amphiphilic molecules at a liquid-liquid interface," Mol. Phys. 59(2), 227-239 (1986).

${ }^{34}$ A. Bymaster and W. G. Chapman, "AniSAFT density functional theory for associating polyatomic molecules," J. Phys. Chem. B 114(38), 12298-12307 (2010).

${ }^{35}$ D. A. McQuarrie, Statistical Mechanics (University Science Books, 2000).

${ }^{36}$ Y. Rosenfeld, "Free-energy model for the inhomogeneous hard-sphere fluid mixture and density-functional theory of freezing," Phys. Rev. Lett. 63(9), 980 (1989)

${ }^{37}$ J.-P. Hansen and I. R. McDonald, Theory of Simple Liquids (Elsevier, 1990).

${ }^{38}$ M. S. Wertheim, "Fluids with highly directional attractive forces. I. Statistical thermodynamics," J. Stat. Phys. 35(1-2), 19-34 (1984).

${ }^{39}$ M. S. Wertheim, "Fluids with highly directional attractive forces. II. Thermodynamic perturbation theory and integral equations," J. Stat. Phys. 35(1-2), 35-47 (1984).

${ }^{40}$ M. S. Wertheim, "Fluids with highly directional attractive forces. III. Multiple attraction sites," J. Stat. Phys. 42(3-4), 459-476 (1986).

${ }^{41}$ M. S. Wertheim, "Fluids with highly directional attractive forces. IV. Equilibrium polymerization,” J. Stat. Phys. 42(3-4), 477-492 (1986).

${ }^{42}$ C. J. Segura, W. G. Chapman, and K. P. Shukla, "Associating fluids with four bonding sites against a hard wall: Density functional theory," Mol. Phys. 90(5), 759-772 (1997).

${ }^{43}$ W. G. Chapman, G. Jackson, and K. E. Gubbins, "Phase equilibria of associating fluids: Chain molecules with multiple bonding sites," Mol. Phys. 65(5), 1057-1079 (1988).

${ }^{44}$ B. D. Marshall, C. Emborsky, K. Cox, and W. G. Chapman, "Effect of bond rigidity and molecular structure on the self-assembly of amphiphilic molecules using second-order classical density functional theory," J. Phys. Chem. B 116(9), 2730-2738 (2012).

${ }^{45}$ E. W. Lemmon, M. L. Huber, and M. O. McLinden, NIST reference fluid thermodynamic and transport properties-REFPROP, 2002.

${ }^{46}$ C. Marche, C. Ferronato, and J. Jose, "Solubilities of n-alkanes (C6 to C8) in water from 30 C to 180 C," J. Chem. Eng. Data 48(4), 967-971 (2003).

${ }^{47}$ P. C. Hiemenz and R. Rajagopalan, Principles of Colloid and Surface Chemistry, Revised and Expanded (CRC Press, 1997), Vol. 14.
${ }^{48}$ M. Velinova, D. Sengupta, A. V. Tadjer, and S.-J. Marrink, "Sphere-to-rod transitions of nonionic surfactant micelles in aqueous solution modeled by molecular dynamics simulations," Langmuir 27(23), 14071-14077 (2011).

${ }^{49}$ L. Huynh, C. Neale, R. Pomès, and C. Allen, "Systematic design of unimolecular star copolymer micelles using molecular dynamics simulations," Soft Matter 6(21), 5491-5501 (2010).

${ }^{50}$ J. B. Hayter and M. Zulauf, "Attractive interactions in critical scattering from non-ionic micelles," Colloid Polym. Sci. 260(11), 1023-1028 (1982).

${ }^{51}$ O. Glatter, G. Fritz, H. Lindner, J. Brunner-Popela, R. Mittelbach, R. Strey, and S. U. Egelhaaf, "Nonionic micelles near the critical point: Micellar growth and attractive interaction," Langmuir 16(23), 8692-8701 (2000).

${ }^{52}$ F. Palazzesi, M. Calvaresi, and F. Zerbetto, "A molecular dynamics investigation of structure and dynamics of SDS and SDBS micelles," Soft Matter 7(19), 9148-9156 (2011).

${ }^{53}$ L.-J. Chen, S.-Y. Lin, C.-C. Huang, and En-M. Chen, "Temperature dependence of critical micelle concentration of polyoxyethylenated non-ionic surfactants," Colloids Surf., A 135(1), 175-181 (1998).

${ }^{54}$ K. Meguro, Y. Takasawa, N. Kawahashi, Y. Tabata, and M. Ueno, "Micellar properties of a series of octaethyleneglycol-n-alkyl ethers with homogeneous ethylene oxide chain and their temperature dependence," J. Colloid Interface Sci. 83(1), 50-56 (1981).

${ }^{55}$ W. Brown, Z. Pu, and R. Rymden, "Size and shape of nonionic amphiphile micelles: NMR self-diffusion and static and quasi-elastic light-scattering measurements on C12E5, C12E7, and C12E8 in aqueous solution," J. Phys. Chem. 92(21), 6086-6094 (1988).

${ }^{56}$ R. G. Alargova, I. I. Kochijashky, M. L. Sierra, and R. Zana, "Micelle aggregation numbers of surfactants in aqueous solutions: A comparison between the results from steady-state and time-resolved fluorescence quenching," Langmuir 14(19), 5412-5418 (1998).

${ }^{57}$ R. Nagarajan, "Molecular packing parameter and surfactant self-assembly: The neglected role of the surfactant tail," Langmuir 18(1), 31-38 (2002).

${ }^{58}$ S.-Y. Lin, Y.-Y. Lin, E.-M. Chen, C.-T. Hsu, and C.-C. Kwan, "A study of the equilibrium surface tension and the critical micelle concentration of mixed surfactant solutions," Langmuir 15(13), 4370-4376 (1999).

${ }^{59}$ B. V. Zhmud, F. Tiberg, and J. Kizling, "Dynamic surface tension in concentrated solutions of $\mathrm{C}_{\mathrm{n}} \mathrm{E}_{\mathrm{m}}$ surfactants: A comparison between the theory and experiment," Langmuir 16(6), 2557-2565 (2000).

${ }^{60} \mathrm{~J}$. Traube, "Ueber die capillaritätsconstanten organischer stoffe in wässerigen lösungen," Justus Liebigs Ann. Chem. 265(1), 27-55 (1891).

${ }^{61}$ M. Kahlweit, R. Strey, and G. Busse, "Microemulsions: A qualitative thermodynamic approach," J. Phys. Chem. 94(10), 3881-3894 (1990).

${ }^{62}$ W. Kunz, F. Testard, and T. Zemb, "Correspondence between curvature, packing parameter, and hydrophilic-lipophilic deviation scales around the phase-inversion temperature," Langmuir 25(1), 112-115 (2008).

${ }^{63}$ R. R. Balmbra, J. S. Clunie, J. M. Corkill, and J. F. Goodman, "Variations in the micelle size of non-ionic detergents," Trans. Faraday Soc. 60, 979-985 (1964).

${ }^{64}$ P. G. Nilsson and B. Lindman, "Water self-diffusion in nonionic surfactant solutions. Hydration and obstruction effects," J. Phys. Chem. 87(23), 4756-4761 (1983).

${ }^{65}$ A. Haghmoradi, L. Wang, and W. G. Chapman, "A density functional theory for association of fluid molecules with a functionalized surface: Fluid-wall single and double bonding," J. Phys.: Condens. Matter 29(4), 044002 (2016).

${ }^{66}$ A. Haghmoradi, L. Wang, and W. G. Chapman, "A density functional theory for colloids with two multiple bonding associating sites," J. Phys.: Condens. Matter 28(24), 244009 (2016).

${ }^{67}$ W. A. Fouad et al., "Extensions of the SAFT model for complex association in the bulk and interface," Fluid Phase Equilib. 416, 62-71 (2016). 